\title{
The effect of conspecific cues on honey bee foraging behavior
}

\author{
Eva S. Horna Lowell ${ }^{1}$, Julie A. Morris ${ }^{1}$, Mayra C. VIDAL ${ }^{2}$, Catherine S. Durso ${ }^{3}$, \\ Shannon M. MurPHY ${ }^{1}$ \\ ${ }^{1}$ Department of Biological Sciences, University of Denver, 2190 E. Iliff Ave, Olin Hall 102, Denver, CO 80210, USA \\ ${ }^{2}$ Department of Biology, 358 Life Sciences Complex, Syracuse University, Syracuse, NY, USA \\ ${ }^{3}$ Center for Statistics and Visualization, University of Denver, Denver, CO, USA
}

Received 29 August 2018 - Revised 9 April 2019 - Accepted 3 June 2019

\begin{abstract}
Foraging honey bees (Apis mellifera) seem to use the presence of conspecific foragers as cues for flower quality. However, there is disagreement regarding how a conspecific cue is perceived by other foragers (enhancement or inhibition). Most studies manipulate the total number of bees foraging in an arena or the presence or absence of a bee on a flower and then observe the behavior of one forager in response to a single conspecific, which does not reflect natural foraging. We tested how a range of conspecifics on flowers affected on which flowers foraging honey bees landed. We trained students from a biology class for non-STEM majors to collect data and tested whether the number of conspecifics on flowers influences on which flower foragers land. We found that foragers land more frequently on flowers occupied by more conspecifics, which supports the hypothesis that conspecifics are cues for local enhancement. Our results increase our understanding of how honey bees forage once at a flower patch.
\end{abstract}

\section{foraging / behavior / honey bee / Apis mellifera / floral resource / social cue}

\section{INTRODUCTION}

Humans depend on honey bees (Apis mellifera) for food production; honey bees are responsible for pollinating 12 billion dollars of crops each year (Calderone 2012). Human dependence on honey bees for major global crop production has led to extensive research regarding the behavior of foraging bees in flower patches within a landscape (Ribbands 1949; von Frisch 1967; Visscher and Seeley 1982; Seeley 1986; Seeley et al. 1991; Beekman and Ratnieks 2000; Sumpter and Pratt 2003). Honey bees are eusocial insects (Wilson

Electronic supplementary material The online version of this article (https://doi.org/10.1007/s13592-019-00657-0) contains supplementary material, which is available to authorized users.

Corresponding author: E. Horna Lowell, evasofiahl@gmail.com

Manuscript editor: James Nieh
1975 ) and use a variety of signals to communicate and coordinate effective nectar and pollen collection among individuals within a hive and to influence honey bee recruitment to a resource patch (e.g., waggle dancing; Pastor and Seeley 2005). Communication signals that occur within a hive and that affect recruitment to a resource patch have been studied relatively extensively (e.g., von Frisch, 1967). However, the ways that honey bees use cues, specifically conspecific cues, to decide which flowers to land and forage upon once at a flower patch are not as well understood.

Foraging honey bees seem to use the presence of conspecifics as a cue to indicate flower quality, but there are very few papers in the literature about this topic and there is disagreement regarding how a conspecific cue is perceived. Two studies have found that conspecifics are a cue for local enhancement for foraging honey bees; the presence of a conspecific forager on a flower attracts 
incoming foragers to that particular flower (Kalmus and Ribbands 1952; Kalmus 1954). Studies on bumblebees have found that conspecifics can benefit from local enhancement cues because the information provided by the conspecific cue regarding the resource is likely to be of high quality and because foragers can reduce the time dedicated to finding food and decisionmaking (Kawaguchi et al. 2006; Avarguès-Weber and Chittka 2014). Only one study has found that conspecifics are cues for local inhibition for foraging honey bees and that the presence of a conspecific forager on a flower deters incoming foragers to that particular flower (Yokoi and Fujisaki 2011). However, Yokoi and Fujisaki (2011) used a dead bee that they pinned onto a flower as the conspecific cue, whereas the studies that found local enhancement used live bees as cues. Dead bees can be used to test visual cues as they resemble live bees but lack chemical cues of live bees; for example, the pheromones deposited by bees after visiting a flower would not be deposited by a dead bee (e.g., Yokoi and Fujisaki 2011 used frozen and dried bees). However, the effectiveness of using dead bees to test for visual cues alone depends on how they were killed; for example, bees that were killed by crushing or preserved in ethanol have other scents that can influence experimental results. Notably, dead bees can be perceived as a signal of danger and thus repel incoming foraging bees (e.g., Dukas 2001). Furthermore, dead bees also do not accurately portray foraging conditions in nature, as live foragers are constantly landing on and taking off of flowers. The study that found local inhibition observed the behavior of a single forager at a time in response to a single conspecific cue (Yokoi and Fujisaki 2011), and the studies that found local enhancement used two to five petri dishes as flowers in their experimental design (Kalmus and Ribbands 1952; Kalmus 1954), instead of a more complex flower array. Honey bees often forage in large fields with a variety of floral resources. By controlling for the number of honey bees that are present on the flowers and the number of flowers presented to the bees, as well as using dead bees, these experiments may not accurately represent how honey bees forage in their natural habitat. Additionally, these studies were not designed to test whether honey bees continued to land on flowers as the number of conspecific cues on the flowers increased, or if honey bees reached a threshold above which they began to avoid landing on flowers where there were too many conspecifics. It is possible that if there are too many conspecifics on a flower, then this could result in competition for nectar resources, deterring incoming foragers from landing on that flower.

We tested whether honey bees use the presence of conspecifics on flowers as cues for local enhancement, improving on prior experiments by not physically manipulating the conspecifics on flowers or the honey bee foragers visiting the flowers. We used a large flower array instead of a limited number of flowers, and we tested how a large range of conspecifics on flowers affected on which flowers foraging honey bees landed. If conspecifics on flowers are cues for local enhancement, then incoming foragers should land on flowers occupied by more conspecifics rather than unoccupied flowers or flowers with fewer bees. Alternatively, if conspecifics on flowers are cues for local inhibition, then honey bees should land on unoccupied flowers or flowers with few bees. If honey bees do not use conspecifics as cues on flowers, then there would be no relationship between the number of bees on a flower and how many bees land on that flower. Our experimental design also allowed us to test whether enhancement continues to occur as the number of bees increases on a flower or whether there is a threshold above which conspecifics no longer serve as enhancement cues. We provided the foraging honey bees with a large flower array, allowed bees to naturally land on the array, and then counted how many bees visited and landed on focal flowers with variable numbers of initial bees already present on that flower. We worked with undergraduate students from a biology course for non-STEM majors at our university who participated in data collection. Their participation allowed us to collect a large data set and also to expose a diverse group of non-scientists to an authentic research experience that could impact their understanding and appreciation for the process of scientific research. 


\section{METHODS}

\subsection{Study system}

We studied honey bees from a hive in a community garden on the campus of our university in August and September of 2016. We created two flower arrays, each with 25 fake flowers that were numbered 1 through 25 , with some flowers located on the edge and some flowers located in the interior of the array (Figure 1). To make the flowers, we used a 2.0-mL Eppendorf tube (with tick markings for every $0.25 \mathrm{~mL}$ ) that we filled with sugar water (1 kg sucrose: $1 \mathrm{~L}$ water) and taped to a $25-\mathrm{cm}$ wooden skewer (the flower "stem"); we then placed a laminated blue square $\left(5 \mathrm{~cm}^{2}\right)$ paper "flower" around the Eppendorf tube. To create each array, we inserted 25 flower skewers into a base made of cardboard and Styrofoam taped together. The horizontal surface of the blue flowers stood $17 \mathrm{~cm}$ above the cardboard, and the edges of the flowers were $10 \mathrm{~cm}$ apart in every direction. During our replicates, we placed the two flower arrays at least $5 \mathrm{~m}$ away from each other and 5-10 $\mathrm{m}$ from the hive. For each data collection period, we haphazardly rotated the position and direction of the flower arrays.

\subsection{Experimental design}

To determine how long each replicate should be for the experiment, we first observed how long it takes for honey bees to deplete the sugar water from the Eppendorf tubes in our flowers to the point where bees would have to compete for access to sugar water $(<1.75 \mathrm{~mL}$ of sugar water in the Eppendorf). When the sugar solution drops below $1.75 \mathrm{~mL}$, only one or two honey bees can crawl inside the tube and access the sugar water at a time, whereas when the Eppendorf tube is full, many more bees can access the sugar water at the same time. We wanted the Eppendorf tubes to remain full during our experiment to ensure that the bees' behavior was due to the initial number of bees on the individual flowers, not as a result of intraspecific competition to reach the sugar water inside the Eppendorf tube. We measured how much the bees depleted a small flower array ( 8 flowers) during 6 different time periods $(0.5,1$,
$1.5,2,3$, and $4 \mathrm{~min}$ ), which we replicated in a random order 13 times. Using a one-way ANOVA, we found that there was a significant difference in how depleted the fake flowers were among the different time periods $\left(F_{5,616}=37.8\right.$, $P<0.001)$ and that flowers were depleted below $1.75 \mathrm{~mL}$ only in the replicates that lasted $4 \mathrm{~min}$ (Figure 3 in the Appendix). Given these results, we made our replicates last $50 \mathrm{~s}$ during our experiment.

To test if a greater number of bees on a flower acted as a cue for enhancement or inhibition for other bees to visit that flower, we counted how many bees landed on flowers with different numbers of initial bees. For 1 week in August 2016, we performed the experiment with members of our lab to ensure that the experimental protocol was clear and precise. We then trained eight different laboratory sections (160 students) from a non-majors biology course to perform our experiment at least three different times before they began collecting data during 1 week in September 2016. Each laboratory section had approximately 20 students and we assigned a maximum of 10 students per flower array. For each replicate, there were two people in charge of filling Eppendorf tubes with sugar water (fillers), at least two people observing flowers (observers, and they observed their assigned focal flower), and one person recording data and keeping time (recorder). If any student was unable to accurately count the number of bees on their focal flower, we removed their data before we analyzed the data $(n=38$ data points out of 1406). During training, we observed the students and counted the bees on their focal flower without their knowledge, thereby determining which students were unable to accurately count bees on a flower; most of these students were assigned to other jobs (e.g., fillers), but we were not always able to do this in a single laboratory section so we removed their data from the final data set. Before each replicate began, the two fillers used dropper pipettes to quickly fill the depleted Eppendorf tubes with sugar water until a bubble formed at the top of the Eppendorf tube for all of the flowers in the array. The recorder assigned each observer to a specific numbered focal flower to watch using a random number generator. Then, the replicate began and observers 


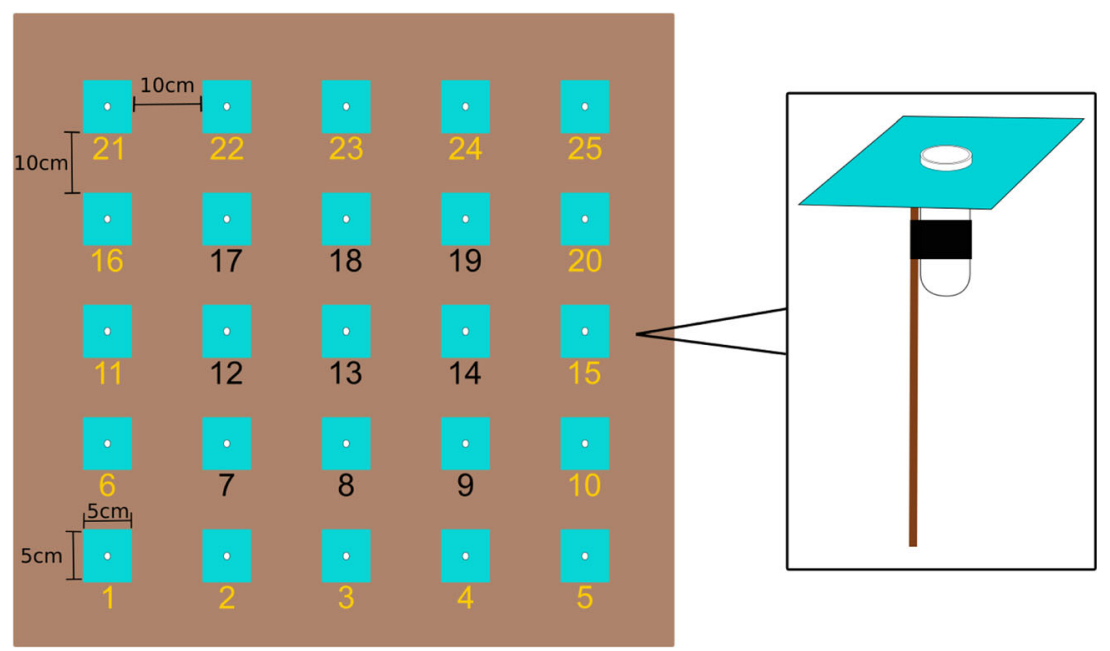

Figure 1. Scheme of one of the flower arrays that we used in the experiment. A total of 25 fake flowers (blue squares) were inserted into a cardboard base. Edge flowers are numbered in yellow and interior flowers are numbered in black (different colors in figure only). The magnified image is of one of the fake flowers that we used in the flower arrays. Each flower has a 2-mL Eppendorf tube inserted into the middle of a square piece of blue laminated construction paper that was then taped to a wooden skewer.

spent exactly $30 \mathrm{~s}$ watching their focal flower, counted the number of bees present on that flower, and then told the recorder the number of bees that were on the flower at the 30-s mark; this value served as the initial number of bees on the focal flower. For the next $20 \mathrm{~s}$, the observers used hand counters to count how many bees landed on the top of their focal flower; we used hand counters so that observers could both report how many bees had been on their flower at the 30-s mark and also continue to count how many bees visited the flower during the remaining 20 -s period. The number of bees that landed on the top of their focal flower during the $20 \mathrm{~s}$ served as the number of bees that visited that flower. Any bees that landed on the wooden stem or underside of the flower were not counted. In order to be able to attribute the observed behavior of foraging bees to the initial number of bees on the focal flower, we limited the amount of time to count incoming foragers to $20 \mathrm{~s}$. After each replicate, the fillers quickly filled the depleted Eppendorf tubes with sugar water again, and then the next replicate began as soon as the fillers moved away from the array. Each laboratory section completed a mean of 30 replicates (range 23-56 replicates).
Additionally, at the beginning and end of each data collection period, we recorded the temperature, time of day, percent humidity, and percent cloud cover, which are all variables known to affect bee foraging.

\subsection{Statistical analysis}

To analyze if bees land on flowers that are occupied by conspecifics more often than flowers occupied by few or no conspecifics (local enhancement), we used a linear mixed effect model with \# bees that visited the focal flower as our response variable and initial \# of bees on focal flower as the independent variable. We standardized our counts of number of bees per replicate to account for variable levels of foraging activity on our flower arrays during the different replicates. For example, in periods of high foraging activity, we may have counted more bees visiting the focal flower simply because there were more bees on the array or fewer bees visiting the focal flower during periods of low foraging activity. To account for this level of variability among replicates, we 
standardized the values for initial \# of bees on focal flower and \# bees that visited the focal flower by incorporating the mean number of bees on all flowers for that replicate with the equation:

(number of bees)-(mean number of bees for that replicate) (standard deviation for that replicate)

Because we standardized the number of bees per replicate, we did not need to include replicate, class, or board in our statistical model. In our final model, our two fixed effects were initial \# of bees on focal flower and flower position (edge or interior), and our random effect was recorder. We originally included as fixed effects into our model two principal components (PCA1 and PCA2) that summarized our climatic variables, which were percent cloud cover, percent humidity, temperature, and time of day; together our principle components summarized $84.6 \%$ of the variation among these variables $(\mathrm{PCA} 1=65.2 \%, \mathrm{PCA} 2=19.4 \%$; Figure 4 in the Appendix). However, the likelihood ratio tests from model comparisons allowed omission of both PCA1 and PCA2 as fixed effects from our final model. Since the likelihood ratio test $P$ value is biased downward, we performed a bootstrap simulation to confirm the significance found using the likelihood ratio test and we report both $P$ values. To perform our models, we used the package lme4 (Bates et al. 2015) with the function lmer in $\mathrm{R}$ environment 3.4.1 ( $\mathrm{R}$ Core Team 2011).

To test whether enhancement continues to occur as we increase the number of bees as cues on a flower, or whether there is a threshold at which the enhancement pattern disappears, we fit our data using a generalized additive model (GAM). We performed our GAM using the package mgcv with the function gam (Wood 2011) in $\mathrm{R}$ environment 3.4.1. In our final model, the \# bees that visited the focal flower was the response variable, initial \# of bees on focal flower was the smooth term, and recorder was included as a penalized regression term (function "bs $=$ re"). We did not include the flower position because the final model without this variable had a better fit. We evaluate the shape of the slope by plotting the final model following Zuur et al. (2009).

Data availabilityData from this study are archived in the Dryad Digital 53 Repository: doi: https://doi.org/10.5061/dryad.3rn48cm (Horna Lowell et al. 2019).

\section{RESULTS}

We found that the initial number of bees on a flower had a significant positive effect on the number of bees that landed on a flower $(t=$ 4.76, $n=1406, P<0.001$, bootstrap $P<0.01$, Figure 2), which means that foraging honey bees were more likely to visit and land on flowers that were occupied by a greater number of conspecific bees. Flower position (edge vs. interior) also significantly affected the number of bees that landed on a flower $(t=$ 1.99, $n=1406, P<0.05$, bootstrap $P<0.05)$; bees were more likely to land on an edge flower than an interior flower. We found that the reported number of bees that landed on a flower differed by recorder (the person recording the data; bootstrap $P<0.01$ ). Our analysis using GAMs yielded the same results that the number of bees landing on a flower increased linearly with the number of bees already present on the focal flower. We found no threshold above which bees stopped visiting an already crowded flower $\left(F_{1,58.26}=334.19, P<\right.$ 0.0001; Figure 5 in the Appendix).

\section{DISCUSSION}

We found that there was a linear relationship between the number of honey bees present on a flower and the number of bees that landed on that same flower, which indicates that incoming foragers land more frequently on flowers with more conspecifics than on flowers with few or no conspecifics. Furthermore, we found that there was no threshold above which honey bees 


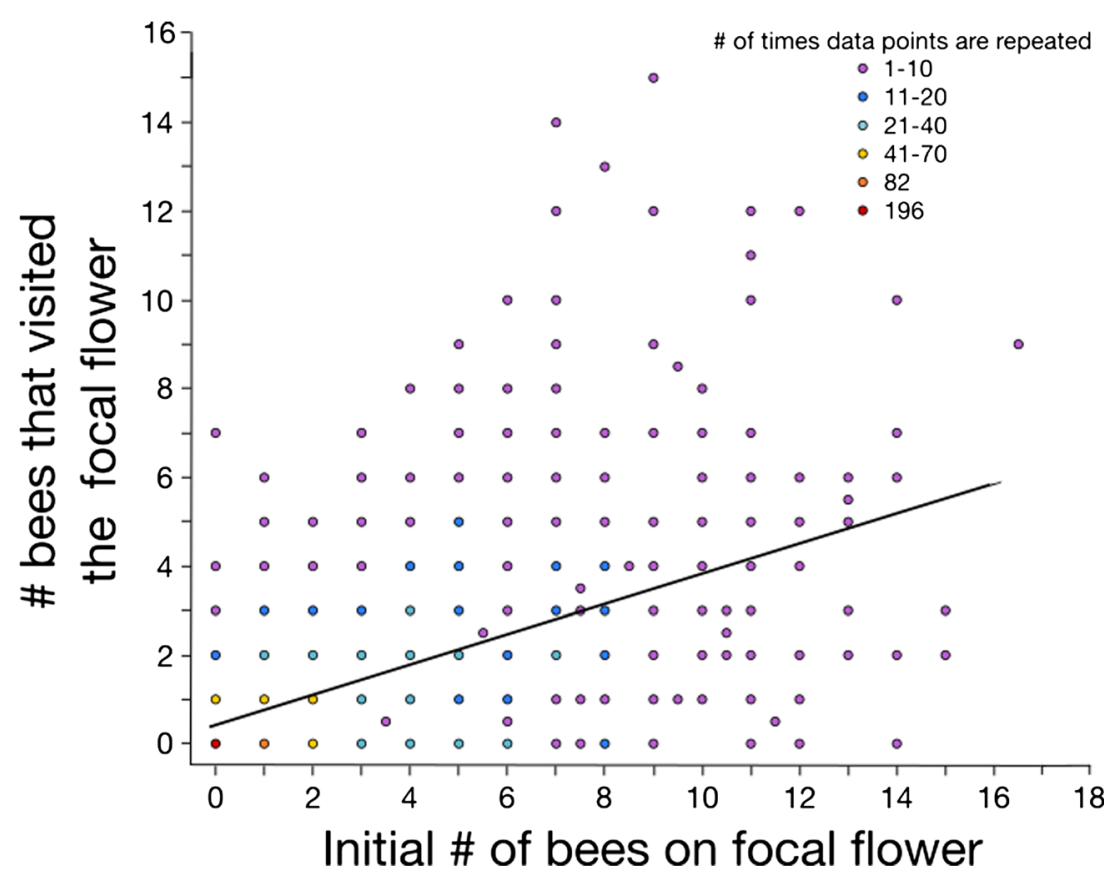

Figure 2. The linear relationship between the initial number of bees on a focal flower and the number of bees that visited that particular flower in $20 \mathrm{~s}\left(y=0.32 x+0.56 ; R^{2}=0.25, P<0.001\right)$.

began to avoid landing on flowers where there were too many conspecifics. Our results support the hypothesis that honey bees use conspecifics as cues that lead to the effect of local enhancement, which is what Kalmus and Ribbands (1952) and Kalmus (1954) also found. Furthermore, our results enhance research in this field because we are the first to have performed an experiment in which we did not physically manipulate the foraging bees and we also had up to 17 foraging bees as our initial number of bees on a flower that incoming foragers could use as cues. Previous experiments have used only a single honey bee as the conspecific cue (dead or alive) and tested the response of a single forager at a time, or tested a limited number of flowers, none of which represent realistic foraging conditions that bees normally encounter.

There are several explanations for why foraging bees visited flowers where there were already foraging conspecifics present more often than flowers that were occupied by few or no conspecifics. One possible aspect that could explain the observed behavior is the ecology of fear (e.g., Bolnick and Preisser 2005; Preisser et al. 2005). Perhaps the presence of conspecifics on a flower suggests a low risk of attack by predators to other foraging honey bees. Dukas (2001) studied flowers used as hunting grounds by crab spiders and found that bees can perceive when a flower is dangerous by the visual cue of the spider itself or of bee corpses. Thus, the presence of live foraging honey bees may be perceived as a safe space for other foraging bees. Local enhancement cues can also lead to crowded foraging groups, and the high density of foragers may be used as a defense mechanism so that other bee species do not steal the floral resource (Slaa et al. 2003).

An important mechanism used by honey bees to perceive conspecifics that could lead to local enhancement is chemical communication (Ferguson and Free 1979; Free and Williams 1983; Stout and Goulson2001). Honey bee foragers deposit pheromones on flowers to communicate with conspecifics the location 
of flowers that are of particularly high quality; flowers with high concentrations of pheromones indicate to other conspecific foragers the high quality of that particular flower (Ferguson and Free 1979; Free and Williams 1983; Stout and Goulson 2001). Thus, the presence of conspecifics on flowers signifies that the flower is a profitable and reliable food source. Another mechanism is communication through dance and other movements of bees. Tautz and Sandeman (2003) showed that when honey bee foragers are trained to visit a highquality, localized resource (i.e., a feeder), the trained foragers would synchronize their flights from the hive so that they arrived in groups with new recruits to the feeder. Upon arrival at the feeder, the trained foragers would fly in a circular motion above the feeder before landing, thereby indicating rewarding resources to other foragers. Furthermore, Tautz and Sandeman (2003) propose that the new recruits watch where the trained foragers land to find a high-quality resource, which may also explain why our foragers landed on flowers occupied by high numbers of conspecifics. Thus, our research supports previous research (Tautz and Sandeman 2003; Riley et al. 2005) that also found that foragers make fine-scale choices using visual or olfactory cues to complete their flights and land on a high-quality flower. Considering these complex communication signals of foraging bees, it is important to allow bees to forage in groups naturally, as we did in our experiment, to measure the response of bees to food sources and to the presence of conspecifics.

We used a large flower array in our experiment and we found that honey bees visited edge flowers more frequently than interior flowers. Edge effects can affect insect behavior (Reeve and Cronin 2010), but generally these habitat edges are on a much larger scale than the $10 \mathrm{~cm}$ that separated our edge and interior flowers (Murphy et al. 2016; Ries et al. 2017 and references within). In our experiment, honey bee foragers may visit the edge flowers more frequently because they are the first flowers they encountered as they approached the array.
A recent study demonstrated that honey bees understand the number zero (Howard et al. 2018). Not only can honey bees understand that a lack of stimulus corresponds to the number zero but they can also understand that the number zero lies on the lower end of the numerical continuum (Howard et al. 2018). Our results are consistent with these findings because we found that honey bees were able to distinguish unoccupied flowers (flowers with no visual stimulus or cues from bees) from flowers that were occupied by honey bees. Furthermore, our honey bees were able to distinguish between low and high numbers, as the foragers consistently landed on flowers with more bees more often than flowers with fewer bees.

This research is an example of our ongoing efforts to provide authentic course-based undergraduate research experiences (CUREs) for a diverse group of non-STEM students. These experiments allowed our students to engage with a novel research question and to participate in the iterative nature of data collection, while simultaneously teaching them about the importance of pollinators in our natural and agricultural systems. In turn, these students helped us collect a large data set within a single week, which would have otherwise taken us a lot longer to collect. These types of activities have been recognized as important components of successful CUREs (Auchincloss et al. 2014; Bangera and Brownell 2014; Ballen et al. 2017), and we are currently assessing the impact of this work on students' performance and attitudes about science.

While our experimental design reduced the extent to which we manipulated the foraging honey bees, there are other biases associated with our design that need to be considered. Before we started our experiment, we trained the foraging honey bees to recruit to our fake flower arrays. Therefore, it is probable that when we were performing the experiment, the foragers visiting our flowers had previous experience foraging on our flower arrays. When we counted the number of new foragers that landed on the focal flower, we did not control for the previous foraging experience of the 
bees that visited the focal flower. The honey bees we counted could have been bees that had previously visited our flower arrays or naïve bees that never had prior experience foraging on our flower arrays. Because we did not control for the previous foraging experience of the honey bees, an experienced bee could have returned to the same flower each time she visited the array, irrespective of the presence of other conspecifics. Nonetheless, our inherently different way of testing how conspecific cues affect the foraging behavior of honey bees led to results that are consistent with previous findings (Kalmus and Ribbands 1952; Kalmus 1954; Tautz and Sandeman 2003).

We found that foraging bees use conspecifics as cues for local enhancement. While other studies mostly tested whether a foraging honey bee was attracted to or repelled by a single conspecific on a flower, we tested how a large range of conspecific foragers on a flower affected upon which flowers foraging honey bees decided to land. We also performed our experiment in a community garden that had a great diversity of flowering plant species, predators, and other bee species whereas other studies performed their experiments in very controlled settings. Our method allowed us to study honey bee foraging behavior without manipulating the bees and thus will help us understand what type of foraging behaviors is most common for honey bees in nature.

\section{ACKNOWLEDGMENTS}

We thank the students from the "Sustaining Life" undergraduate class at the University of Denver for helping to collect data and the teaching assistants from all the lab sections of the course who helped the students run the experiment (Faith Lierheimer, Lisa Clark, Claudia Hallagan, and lab director, Angie Hebel). We thank the Murphy lab, the University of Denver Ecology and Evolutionary Biology (DUEEB) group, and Amy Toth for helpful suggestions on earlier versions that greatly improved our manuscript.

\section{AUTHORS' CONTRIBUTIONS}

ESHL and SM conceived the project and designed the experiment. ESHL, SM, and JM trained students to perform the experiment. ESHL, SM, JM, and MV performed data collection. CD and MV performed statistical analysis. ESHL wrote the first draft of the paper, and all authors helped write sections of the paper or contributed substantially to revisions. All authors have read and approved the final version of this paper.

\section{FUNDING INFORMATION}

We thank the University of Denver Undergraduate Research Center for funding our research with an award to ESHL.L'effet des signaux spécifiques des congénères sur le comportement de recherche de nourriture des abeilles mellifères

Butiner / comportement / Apis mellifera / ressource florale / signal sociale

Der Effekt artspezifischer Signale auf das Sammelverhalten von Honigbienen

Sammeln / Verhalten / Honigbiene / Apis mellifera / Blütenresourcen / soziales Signal

\section{REFERENCES}

Auchincloss, L.C., Laursen, S.L., Branchaw, J.L., Eagan, K., Graham, M., et al. (2014) Assessment of coursebased undergraduate research experiences: Ameeting report. CBE Life Sci. Educ., 13, 29-40.

Avarguès-Weber, A. \& Chittka, L. (2014) Local enhancement or stimulus enhancement? Bumblebee social learning results in a specific pattern of flower preference. Anim. Behav., 97, 185-191.

Ballen, C.J., Blum, J.E., Brownell, S., Hebert, S., Hewlett, J., et al (2017) A call to develop course-based undergraduate research experiences (CUREs) for Nonmajors courses. CBE Life Sci. Educ., 16, 1-7.

Bangera, G. \& Brownell, S.E. (2014) Course-based undergraduate research experiences can make scientific research more inclusive. CBE Life Sci. Educ., 13, 602606. 
Bates, D., Machler, M., Bolker, B. \& Walkter, S. (2015) Fitting linear mixed-effects models using lme4. J. Stat. Softw., 1-48.

Beekman, M. \& Ratnieks, F.L.W. (2000) Long-range foraging by the honey-bee, Apis mellifera L. Funct. Ecol., 14, 490-496.

Bolnick, D. \& Preisser, E. (2005) Resource Competition Modifies the Strength of Trait-Mediated Predator-Prey Interactions: A Meta Analysis. Ecology, 86, 27712779.

Calderone, N.W. (2012) Insect pollinated crops, insect pollinators and US agriculture: Trend analysis of aggregate data for the period 1992-2009. PLoS One, 7, 24-28.

Dukas, R. (2001) Effects of perceived danger on flower choice by bees. Ecol. Lett., 4, 327-333.

Ferguson, A.W. \& Free, J.B. (1979) Production of a Forage-Marking Pheromone by the Honeybee. J. Apic. Res., 18, 128-135.

Free, J.B. \& Williams, I.H. (1983) Scent-Marking of Flowers by Honeybees. J. Apic. Res., 22, 86-90.

von Frisch, K. (1967) The Dance Language and Orientation of Bees. The Belknap Press of Harvard University Press, Cambridge.

Horna Lowell, E.S., Morris, J.A., Vidal, M.C., Durso, C.S. \& Murphy, S.M. (2019) Data from: The effect of conspecific cues on honey bee foraging behavior. Dyrad Digital Repository. https://doi.org/10.5061/dryad. $3 \mathrm{rn} 48 \mathrm{~cm}$.

Howard, S.R., Garcia, J.E., Greentree, A.D. \& Dyer, A.G. (2018) Numerical ordering of zero in honey bees. Science (80-. )., 360, 1124-1126.

Kalmus, H. (1954) The clustering of honeybees at a food source. Br. J. Anim. Behav., 2, 63-71.

Kalmus, H. \& Ribbands, C.R. (1952) The origin of the odours by which honeybees distinguish their companions. Proc. R. Soc. B Biol. Sci., 140, 50-59.

Kawaguchi, L.G., Ohashi, K. \& Toquenaga, Y. (2006) Do bumble bees save time when choosing novel flowers by following conspecifics? Funct. Ecol., 20, 239-244.

Murphy, S.M., Battocletti, A.H., Tinghitella, R.M., Wimp, G.M. \& Ries, L. (2016) Complex community and evolutionary responses to habitat fragmentation and habitat edges: what can we learn from insect science? Curr. Opin. Insect Sci., 14, 61-65.

Pastor, K.A. \& Seeley, T.D. (2005) The Brief Piping Signal of the Honey Bee: Begging Call or Stop Signal? Ethology, 111, 775-784.

Preisser, E.L., Bolnick, D.I. \& Benard, M.E. (2005) Scared to Death ? The Effects of Intimidation and Consumption in Predator-Prey Interactions Published by : Ecological Society of America SCARED TO DEATH ? THE EFFECTS OF INTIMIDATION AND CONSUMPTION IN PREDATOR-PREY INTERACTIONS. Ecology, 86, 501-509.

R Core Team (2011) R: A language and environment for statistical computing. R Foundation for Statistical
Computing, Vienna. http://www.R-project.org/. Accessed June 2018.

Reeve, J.D. \& Cronin, J.T. (2010) Edge behaviour in a minute parasitic wasp. J. Anim. Ecol., 79, 483-490.

Ribbands, C.R. (1949) The Foraging Method of Individual Honey-Bees. J. Anim. Ecol., 18, 47-66.

Ries, L., Murphy, S.M., Wimp, G.M. \& Fletcher, R.J. Jr (2017) Closing Persistent Gaps in Knowledge About Edge Ecology. Curent Landsc. Ecol. Rep., 2, 30- 41.

Riley, J.R., Greggers, U., Smith, A.D., Reynolds, D.R. \& Menzel, R. (2005) The flight paths of honeybees recruited by the waggle dance. Nature, 435, 205-207.

Seeley, T.D. (1986) Social foraging by honeybees: how colonies allocate foragers among patches of flowers. Behav. Ecol. Sociobiol., 19, 343-354.

Seeley, T.D., Camazine, S. \& Sneyd, J. (1991) Collective decision-making in honey bees: how colonies choose among nectar sources. Behav. Ecol. Sociobiol., 28, 277-290.

Slaa, E.J., Wassenberg, J. \& Biesmeijer, J.C. (2003) The use of field-based social information in eusocial foragers: local enhancement among nestmates and heterospecifics in stingless bees. Ecol. Entomol., 28, 369-379.

Stout, J.C. \& Goulson, D. (2001) The use of conspecific and interspecific scent marks by foraging bumblebees and honeybees. Anim. Behav., 62, 183-189.

Sumpter, D. \& Pratt, S. (2003) A modelling framework for understanding social insect foraging. Behav. Ecol. Sociobiol., 53, 131-144.

Tautz, J. \& Sandeman, D.C. (2003) Recruitment of honeybees to non-scented food sources. J. Comp. Physiol. A, 189, 293-300.

Visscher, P.K. \& Seeley, T.D. (1982) Foraging Strategy of Honeybee Colonies in a Temperate Deciduous Forest. Ecology, 63, 1790-1801.

Wilson, E.O. (1975) Sociobiology: The new Synthesis. In Sociobiology: The New Synthesis. Harvard University Press, Cambridge.

Wood, S.N. (2011) Fast stable restricted maximum likelihood and marginal likelihood estimation of semiparametric generalized linear models. J. R. Stat. Soc. Series B. 73, 3-36.

Yokoi, T. \& Fujisaki, K. (2011) To Forage or Not: Responses of Bees to the Presence of Other Bees on Flowers. Ann. Entomol. Soc. Am., 104, 353-357.

Zuur, A., Ieno, E.N., Walker, N., Saveliev, A.A. \& Smith, G.M. (2009) Mixed effects models and extensions in ecology with R. Springer, Berlin.

Publisher's note Springer Nature remains neutral with regard to jurisdictional claims in published maps and institutional affiliations. 